\title{
Disorder-induced pseudodiffusive transport in graphene nanoribbons
}

\author{
P. Dietl, ${ }^{1}$ G. Metalidis,,${ }^{1,2}$ D. Golubev, ${ }^{1}$ P. San-Jose,${ }^{3}$ E. Prada,${ }^{3}$ H. Schomerus,${ }^{3}$ and G. Schön ${ }^{1,2}$ \\ ${ }^{1}$ Institut für Theoretische Festkörperphysik, Universität Karlsruhe, D-76128 Karlsruhe, Germany \\ ${ }^{2}$ DFG Center for Functional Nanostructures (CFN), Universität Karlsruhe, 76128 Karlsruhe, Germany \\ ${ }^{3}$ Department of Physics, Lancaster University, Lancaster LA1 4YB, United Kingdom
}

(Received 22 April 2009; published 13 May 2009)

\begin{abstract}
We study the transition from ballistic to diffusive and localized transport in graphene nanoribbons in the presence of binary disorder, which can be generated by chemical adsorbates or substitutional doping. We show that the interplay between the induced average doping (arising from the nonzero average of the disorder) and impurity scattering modifies the traditional picture of phase-coherent transport. Close to the Dirac point, intrinsic evanescent modes produced by the impurities dominate transport at short lengths giving rise to a regime analogous to pseudodiffusive transport in clean graphene, but without the requirement of heavily doped contacts. This intrinsic pseudodiffusive regime precedes the traditional ballistic, diffusive, and localized regimes. The last two regimes exhibit a strongly modified effective number of propagating modes and a mean free path which becomes anomalously large close to the Dirac point.
\end{abstract}

DOI: 10.1103/PhysRevB.79.195413

PACS number(s): 72.15.Rn, 71.23.An, 72.15.Lh

\section{INTRODUCTION}

Graphene continues to fascinate, due in particular to its transport properties close to the Dirac point, where a finite conductivity is observed experimentally ${ }^{1}$ in spite of the vanishing density of states. For clean graphene, simple Dirac fermion models ${ }^{2}$ prove to be sufficient to describe this effect based on a gapless spectrum of evanescent modes pinned at the reservoir contacts that allow for quantum tunneling through macroscopically large graphene samples. ${ }^{3,4}$ Surprisingly, the full transport statistics becomes indistinguishable from that of diffusive metals, ${ }^{4}$ for which reason the name "pseudodiffusive" was coined to describe this transport regime. Furthermore, these evanescent modes can give rise to Hanbury Brown-Twiss-type cross correlations in a ballistic multiprobe graphene structure ${ }^{5}$ similar to those observed in conventional diffusive conductors.

Just as ballistic transport, the effects of disorder in graphene display a surprising richness. For example, armchair ribbons are found to be more sensitive to bulk disorder as their zigzag counterparts, while edge disorder can open up transport gaps in both of them. ${ }^{6}$ In general, different types of disorder can be classified in terms of preserved symmetries, including chirality which is preserved for disorder that is smooth on the scale of interatomic distances ${ }^{7-10}$ and significantly modifies the standard results for transport in disordered metals. For example, carriers in graphene ribbons with smooth disorder cannot be localized ${ }^{11}$ and their conductivity is seen to increase with system size $L$ as $\sigma \sim \sqrt{L / l_{m}}$ in onedimensional (1D), ${ }^{12}$ and $\sim \ln L / l_{m}$ in two dimensional (2D), where $l_{m}$ is the mean free path. ${ }^{13,14}$ Short-range disorder, on the other hand, does not preserve chirality and is therefore generally understood to induce a sequence of ballistic transport, diffusion, and Anderson localization in quasi-1D wires of increasing length. ${ }^{9,15,16}$

Until now, the influence of evanescent modes close to the Dirac point has been studied in ballistic systems only, and it is therefore unclear whether their peculiar spectrum could manifest itself in some significant way in the conductivity of disordered graphene nanoribbons. In this work we will show that evanescent modes play a crucial role for the transport in graphene nanoribbons with short-range disorder. In particular, intrinsic evanescent modes arising from the disorder itself (i.e., independently of the contacts) can reinstate an "intrinsic pseudodiffusive" regime which precedes the ballistic, diffusive, and localized regimes. This regime arises if the short-range disorder does not vanish on average and therefore induces systematic doping, as is the case, e.g., for chemical adsorbate ${ }^{17}$ or substitutional doping. ${ }^{18}$ We describe such systems using a binary disorder model (the random binary alloy ${ }^{19,20}$ ) and obtain our results by combining numerical computations with analytical considerations. For longer ribbons, the intrinsic pseudodiffusive regime crosses over into the traditional diffusive and localized regimes, but we find that the mean free path becomes anomalously large at an effective Dirac energy which takes the induced doping into account.

\section{BINARY DISORDER MODEL}

We base our investigation of binary disorder in a graphene nanoribbon on the following tight-binding Hamiltonian,

$$
H=-t \sum_{\langle i, j\rangle} c_{i}^{\dagger} c_{j}+\sum_{i} v_{i} c_{i}^{\dagger} c_{i} .
$$

The first term describes clean graphene with hopping amplitude $t \approx 2.9 \mathrm{eV}$ between nearest neighbors on the hexagonal lattice of carbon atoms (with interatomic distance $a$ $=1.49 \AA$ ). The second term describes binary disorder generated by onsite energies $v_{i}$ which are uncorrelated random variables that take values $v_{\text {high }=V_{0}}$ with probability $p$ and $v_{\text {low }=0}$ with probability $1-p$. We have dropped spin indices in the model since spin is a passive degree of freedom in all our discussion.

Physically, this model describes, for example, a graphene nanoribbon with a fraction $p$ of its sites coupled to identical chemical species adsorbed on its surface. If these adsorbates 


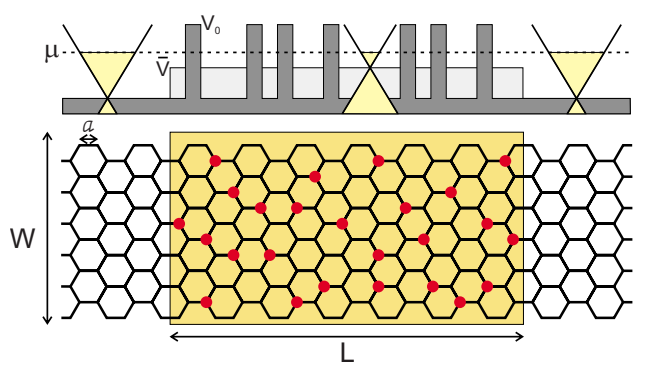

FIG. 1. (Color online) Schematic view of an armchair ribbon with width $W$. Disorder is introduced in a region of length $L$ by adding a potential $V_{0}$ to a percentage $p$ of lattice sites. Disorder sites are depicted by dots. The induced local doping, due to the nonzero average of the disorder, is effectively described by a barrier of height $\bar{V}=p V_{0}$.

are screened their presence is equivalent to a (real and energy dependent) self-energy term acting on each of the carbon sites coupled to an adsorbate. ${ }^{17}$ Typical values of $V_{0}$ can be estimated from density functional theory (DFT) calculations, ${ }^{21}$ and for species such as $\mathrm{Ag}, \mathrm{Cu}$, or $\mathrm{Au}$, one finds values $V_{0} \approx 0.5 \mathrm{eV} \approx 0.2 t$ which are small as compared to $t$.

\section{RESULTS AND DISCUSSION}

To probe the intrinsic transport properties in the presence of binary disorder we present results of numerical computations on nanoribbons of width $W$ and a disordered region of length $L$ with the reservoir contacts placed at $x= \pm \infty$ (see Fig. 1). These computations are based on the recursive Green's-function technique. ${ }^{22-24}$ We obtain qualitatively similar results for metallic and semiconducting armchair ribbons as well as for zigzag ribbons; we therefore only show results for metallic armchair ribbons.

Figure 2 shows the averaged conductance of a ribbon of width $W=103 \sqrt{3} a / 2$ as a function of the length of the disordered region; different curves correspond to different values of the chemical potential. The disorder parameters used, $p$ $=0.4$ and $V_{0}=0.25 t$, correspond to a substantial concentration of adsorbates that are weakly hybridized with their carbon hosts; a sample size of 50 impurity configurations is used in the average.

Two distinct transport regimes immediately catch the eyes. The conductance first decreases sharply over a length scale comparable to the ribbon width $W$ and then abruptly crosses over to a much slower decay. The conductivity $\sigma$ $=\langle G\rangle L / W$ in the pseudodiffusive regime, shown in Fig. 3, develops a clear plateau at the value $\sigma=2 G_{0} / \pi$ (the minimal conductivity in clean graphene ${ }^{3,4}$ ) when the chemical potential $\mu$ coincides with the average value $\bar{V}=p V_{0}$ of the disorder. We now argue that this transport regime indeed constitutes an intrinsic pseudodiffusive regime arising from disorder-induced evanescent modes, while the crossover to the truly diffusive regime is characterized by a mean free path which becomes anomalously large at $\mu=\bar{V}$.

\section{A. Intrinsic pseudodiffusive regime}

To understand the numerical results for short lengths it is useful to compare the average conductance of Fig. 2 to the

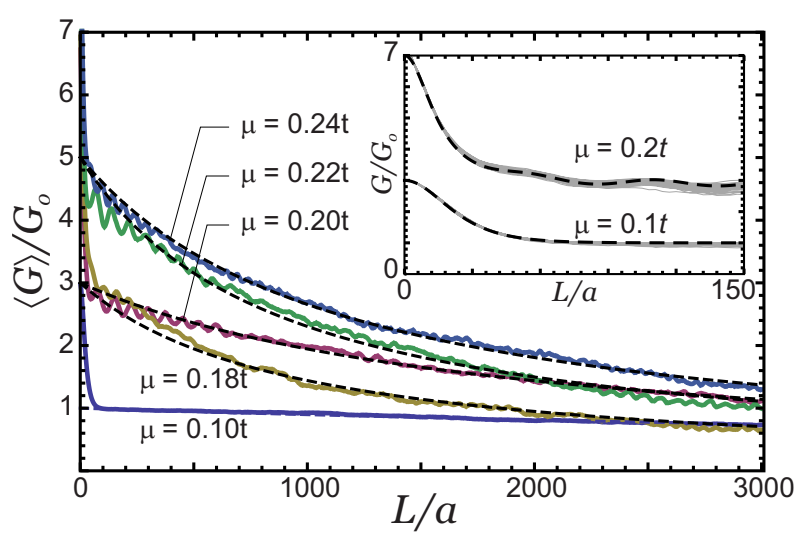

FIG. 2. (Color online) Averaged conductance $\langle G\rangle$ (in units $G_{0}$ $\left.=\frac{2 e^{2}}{h}\right)$ of an armchair ribbon of width $W=103 \sqrt{3} a / 2$ as a function of the length of the disordered region for different chemical potentials. The parameters of the binary disorder are $p=0.4, V_{0}=0.25 t$, so that the effective Dirac point in the disordered region is shifted from zero to $\bar{V}=p V_{0}=0.1 t$. Dashed curves are fits to Eq. (3), with $l_{m}$ as a fit parameter. Inset: pseudodiffusive regime zoom in. Thin gray curves correspond to different disorder realizations. Good agreement is obtained with the ballistic conductance through a square potential barrier of height $\bar{V}$, shown in dashed black, see Eq. (2).

conductance of the same nanoribbon in which the disordered region is replaced by a potential barrier of height equal to the average disorder doping $\bar{V}=p V_{0}$. We compute the conductance through the barrier, $G_{b}$, by wave matching within the Dirac equation description of low-energy transport, which yields

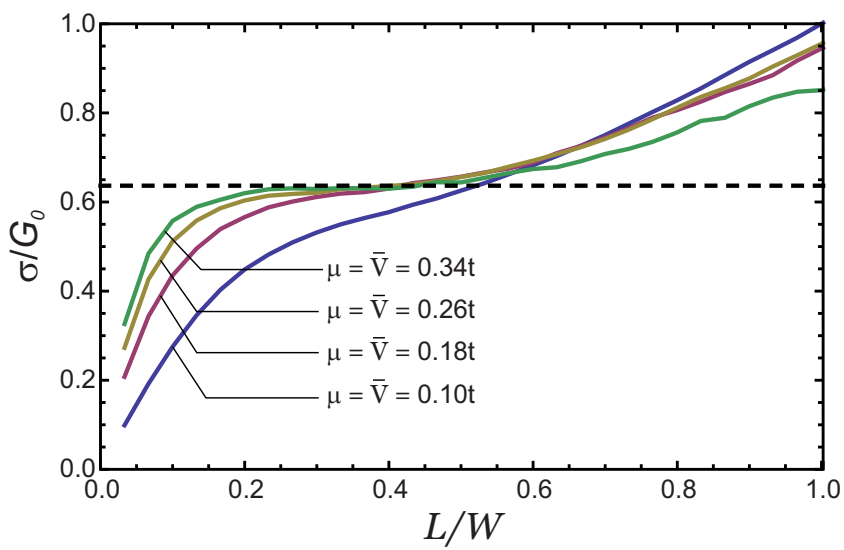

FIG. 3. (Color online) Conductivity $\sigma=\langle G\rangle L / W$ versus length for an armchair ribbon of width $W=103 \sqrt{3} a / 2$ at the effective Dirac point $\mu=\bar{V}$ and fixed impurity fraction $p=0.4$. Different curves correspond to different strength $V_{0}=\bar{V} / p$ of the disorder. As the chemical potential of the effective Dirac point increases, the number $N$ of incoming modes grows, and a plateau develops at the universal conductivity minimum $2 G_{0} / \pi$ (dashed black line). 


$$
G_{b}=G_{0} \sum_{n}\left|\frac{\left(1-z_{n k}\right)^{2}\left(1-z_{n k^{\prime}}\right)^{2}}{e^{i k^{\prime} L}\left(z_{n k}-z_{n k^{\prime}}\right)^{2}+e^{-i k^{\prime} L}\left(1-z_{n k} z_{n k^{\prime}}\right)^{2}}\right|^{2} .
$$

Here the conductance quantum is defined by $G_{0}=2 e^{2} / h$, the sum runs from $n=-(N-1) / 2$ to $(N-1) / 2$, the number of incoming propagating modes is $N=1+2 \operatorname{Int}[\mu W /(\pi \hbar v)], \mu$ is the reservoir chemical potential, the Fermi velocity is given by $v=\frac{3}{2} t a / \hbar, z_{n k}=\left(k+i q_{n}\right) / \sqrt{k^{2}+q_{n}^{2}}, k=\sqrt{(\mu / \hbar v)^{2}-q_{n}^{2}}$, $k^{\prime}=\sqrt{[(\mu-\bar{V}) / \hbar v]^{2}-q_{n}^{2}}$, and $q_{n}=n \pi / W$.

The comparison between $G$ and $G_{b}$ over the range of the abrupt decay is shown in the inset of Fig. 2, where each gray curve corresponds to an individual disorder configuration and the dashed black curve corresponds to Eq. (2). We obtain a remarkably good agreement of both curves even down to hallmark features such as Fabry-Pérot oscillations. This good agreement only begins to break down at lengths for which the disorder starts to mix modes, i.e., the mean free path $l_{m}$, which will be discussed below.

The implications are clear: the sharp decline of conductance observed at lengths $L \leqq W$ is due to the decay of evanescent modes induced by the sudden change in local average potential $\bar{V}$. This average potential shift is well defined because the effect of the disorder is self-averaging at the considered impurity concentrations. Although these evanescent waves are produced by the impurities, their combined effect is equivalent to evanescent modes pinned at the boundary of the disorder region, $x=0$ and $x=L$, which allow tunneling through an effective potential barrier $\bar{V}=p V_{0}$. Furthermore, their contribution to transport at energies $\mu \sim \bar{V}$ and very wide ribbons produces the universal statistics of diffusive metals. Therefore, the observed fast decay in average conductance is the signature of a disorder-induced pseudodiffusive regime. As we will show in Sec. III B, the self-averaging of the disorder arises since the mean free path becomes anomalously large around the effective Dirac point $\mu=\bar{V}$. Contrary to the ballistic regime, the intrinsic pseudodiffusive regime is therefore universal in the limit of wide and short ribbons, which is verified by the negligible spread of the curves in the inset of Fig. 2 over the range of the abrupt decay.

\section{B. Ballistic to diffusive crossover}

At lengths $L \sim W$ evanescent modes through the disordered region have decayed and give way to ballistic propagation of the remaining $N_{p}(\mu)=1+2 \operatorname{Int}[(\mu-\bar{V}) W /(\pi \hbar v)]$ modes up to lengths $L$ comparable to the mean free path $l_{m}$, where the traditional diffusive regime sets in. In the range $W<L<N_{p} l_{m}$, therefore, the large- $N_{p}$ form of the average conductance in the ballistic to diffusive crossover becomes approximately valid, ${ }^{25}$

$$
\langle G\rangle=G_{0} \frac{N_{p}}{1+L / l_{m}} .
$$

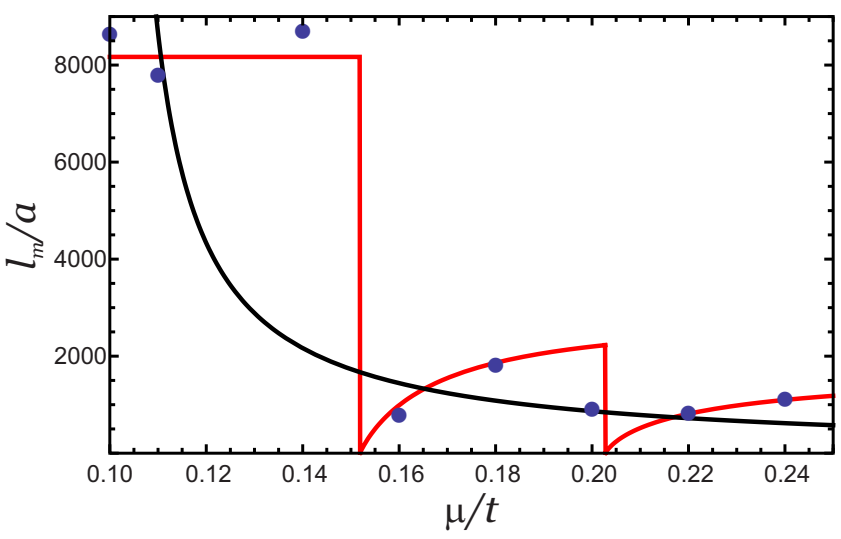

FIG. 4. (Color online) Mean free path as a function of chemical potential for a metallic armchair nanoribbon of width $W$ $=103 \sqrt{3} a / 2$, impurity strength $V_{0}=0.25 t$, and impurity fraction $p$ =0.4. Black curve: bulk value, Eq. (4). Light (red) curve: finite width result, Eq. (5). Dots: values obtained by fitting numerical results to Eq. (3).

This is followed by a transition into a localized regime for nanoribbon lengths greater that the localization length $\xi$ $=N_{p} l_{m}$ for which $\langle G\rangle\left\langle G_{0}\right.$. From that point on, $\langle G\rangle$ decays exponentially as $\exp (-2 L / \xi)$.

The mean free path in the bulk graphene sample is known to be inversely proportional to the electron energy and the disorder strength, see, e.g., Refs. 26 and 27. Adapting the results of these references to our binary disorder model we arrive at the following expression for the mean free path:

$$
l_{m}=\frac{3 \sqrt{3}}{4} \frac{a t^{3}}{p(1-p) V_{0}^{2}|\mu-\bar{V}|} .
$$

The $1 /|\mu-\bar{V}|$ divergence can be traced back to the vanishing bulk density of states of graphene at $\mu \approx \bar{V}$ in the presence of average doping $\bar{V}=p V_{0}$. This dependence is consistent with the behavior observed in Fig. 2 for lengths greater than $W$. However, a detailed analysis shows that Eq. (4) provides only a qualitative description of our data. In order to improve the agreement one has to account for the finite-size effects in the density of states. In the simplest weak disorder approximation the mean free path acquires the form ${ }^{15}$

$$
l_{m}=\frac{\pi \sqrt{3}}{4} \frac{t^{3} W}{p(1-p) V_{0}^{2}} \frac{N_{p}}{\rho^{2}(\mu-\bar{V})},
$$

where the effective density of states $\rho(\mu)$ is defined as follows:

$$
\rho(\mu)=1+2|\mu| \sum_{n=1} \frac{\theta\left(\mu^{2}-E_{n}^{2}\right)}{\sqrt{\mu^{2}-E_{n}^{2}}} .
$$

Here $E_{n}=\hbar v n \pi / W$ are threshold energies at which new channels open up. According to Eq. (5) the mean free path is no longer divergent at $\mu=\bar{V}$, rather it saturates at a finite value $l_{\max } \propto W$. In Fig. 4 we show the comparison of the mean free path Eq. (5) to the one resulting from fitting our 


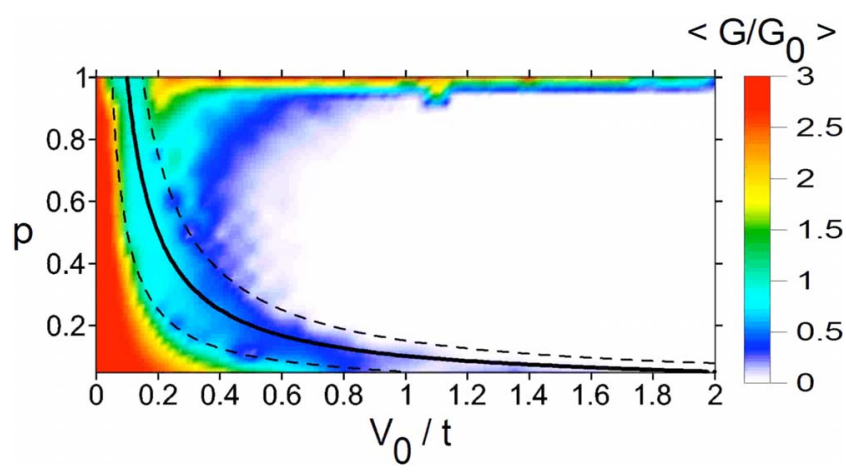

FIG. 5. (Color online) Conductance of a graphene armchair ribbon of length $L=3000 a$ and width $W=103 \sqrt{3} a / 2$ for different values of the impurity fraction $p$ and scattering potential $V_{0}$. The chemical potential is fixed to $\mu=0.1 t$. The solid line corresponds to the condition $\mu=\bar{V}=p V_{0}$. The dotted lines are guides for the eyes, bounding the region of increased conductance around the effective Dirac energy.

data in Fig. 2 with Eq. (3); we find that Eq. (5) reproduces our numerical data very accurately.

\section{Dependence of conductance on disorder parameters}

In order to give a comprehensive overview of the interplay between the various disorder parameters, we have calculated the conductance of a long metallic graphene ribbon $(L=3000 a)$ for different parameters $p$ and $V_{0}$ at a fixed chemical potential $\mu=0.1 t$. The results are summarized in Fig. 5. Parameters $p$ and $V_{0}$ that lead to a large mean free path show up as regions with a relatively high conductance. For example, in the region around $p V_{0}=\mu$ depicted with a solid black curve in Fig. 5, the conductance is close to $G$ $=2 e^{2} / h$, even for this rather long ribbon. The conductance is also large around $p=0$ and $p=1$, which correspond to the regimes where the effective disorder strength $p(1-p) V_{0}^{2}$ is small. This factor thus accounts for the semilunar shape of the phase diagram. Nevertheless, the physical picture leading to a high conductance is somewhat different for small and high disorder percentages. For small $p$ (and $V_{0}$ ), we are in the strict ballistic regime, where the electrons do not feel the impurities at all, not even their average doping effect. On the other hand, for high $p$ we have effectively a disorder-induced potential barrier in the system.

\section{CONCLUSION}

In conclusion, we considered the conductance of graphene nanoribbons containing binary disorder (typical for chemical doping) which induces a shift $\bar{V}$ of the effective Dirac point along with scattering on single discrete impurities. For short ribbons we identified a transport regime dominated by evanescent modes which give rise to an intrinsic form of pseudodiffusive transport. At a longer length scale, a ballistic, and then a diffusive regime is entered where the mean free path is determined both by scattering on the individual impurities as well as by the shifted potential. Close to the effective Dirac point, the mean free path becomes anomalously large, which ensures the universality of the intrinsic pseudodiffusive regime. Compared to ordinary disordered conductors, the presence of nonzero-average short-range disorder typical for chemically doped graphene therefore adds a stage to the conventional path toward localization. The predicted intrinsic pseudodiffusion should be directly measurable in chemically functionalized graphene nanoribbons.

\section{ACKNOWLEDGMENT}

This research was supported by the European Commission via Marie Curie Excellence under Grant No. MEXT-CT2005-02377.
${ }^{1}$ K. Novoselov, A. Geim, S. Morozov, D. Jiang, M. Katsnelson, I. Grigorieva, S. Dubonos, and A. Firsov, Nature (London) 438, 197 (2005).

${ }^{2}$ J. González, F. Guinea, and M. Vozmediano, Nucl. Phys. B 406, 771 (1993).

${ }^{3}$ M. Katsnelson, Eur. Phys. J. B 51, 157 (2006).

${ }^{4}$ J. Tworzydlo, B. Trauzettel, M. Titov, A. Rycerz, and C. W. J. Beenakker, Phys. Rev. Lett. 96, 246802 (2006).

${ }^{5}$ M. A. Laakso and T. T. Heikkilä, Phys. Rev. B 78, 205420 (2008).

${ }^{6}$ E. R. Mucciolo, A. H. Castro Neto, and C. H. Lewenkopf, Phys. Rev. B 79, 075407 (2009).

${ }^{7}$ E. McCann, K. Kechedzhi, V. I. Fal'ko, H. Suzuura, T. Ando, and B. L. Altshuler, Phys. Rev. Lett. 97, 146805 (2006).

${ }^{8}$ I. L. Aleiner and K. B. Efetov, Phys. Rev. Lett. 97, 236801 (2006).

${ }^{9}$ P. M. Ostrovsky, I. V. Gornyi, and A. D. Mirlin, Phys. Rev. B 74, 235443 (2006).

${ }^{10}$ A. Schuessler, P. M. Ostrovsky, I. V. Gornyi, and A. D. Mirlin,
Phys. Rev. B 79, 075405 (2009).

${ }^{11}$ K. Nomura, M. Koshino, and S. Ryu, Phys. Rev. Lett. 99, 146806 (2007).

${ }^{12}$ M. Titov, EPL 79, 17004 (2007).

${ }^{13}$ P. San-Jose, E. Prada, and D. S. Golubev, Phys. Rev. B 76, 195445 (2007).

${ }^{14}$ J. H. Bardarson, J. Tworzydlo, P. W. Brouwer, and C. W. J. Beenakker, Phys. Rev. Lett. 99, 106801 (2007).

${ }^{15}$ A. Cresti, N. Nemec, B. Biel, G. Niebler, F. Triozon, G. Cuniberti, and S. Roche, Nano Res. 1, 361 (2008).

${ }^{16}$ D. A. Areshkin, D. Gunlycke, and C. T. White, Nano Lett. 7, 204 (2007).

${ }^{17}$ J. P. Robinson, H. Schomerus, L. Oroszlany, and V. I. Fal'ko, Phys. Rev. Lett. 101, 196803 (2008).

${ }^{18}$ A. Lherbier, X. Blase, Y.-M. Niquet, F. Triozon, and S. Roche, Phys. Rev. Lett. 101, 036808 (2008).

${ }^{19}$ M. M. Pant and B. Y. Tong, J. Phys. C 13, 1237 (1980).

${ }^{20}$ D. H. Dunlap, H.-L. Wu, and P. W. Phillips, Phys. Rev. Lett. 65, 88 (1990). 
${ }^{21}$ G. Giovannetti, P. A. Khomyakov, G. Brocks, V. M. Karpan, J. van den Brink, and P. J. Kelly, Phys. Rev. Lett. 101, 026803 (2008).

${ }^{22}$ G. Metalidis and P. Bruno, Phys. Rev. B 72, 235304 (2005).

${ }^{23}$ S. Sanvito, C. J. Lambert, J. H. Jefferson, and A. M. Bratkovsky, Phys. Rev. B 59, 11936 (1999).
${ }^{24}$ J. P. Robinson and H. Schomerus, Phys. Rev. B 76, 115430 (2007).

${ }^{25}$ C. W. J. Beenakker, Rev. Mod. Phys. 69, 731 (1997).

${ }^{26}$ N. Shon and T. Ando, J. Phys. Soc. Jpn. 67, 2421 (1998).

${ }^{27}$ H. Suzuura and T. Ando, Phys. Rev. Lett. 89, 266603 (2002). 